\title{
Análogo mecânico para condutividade elétrica dos metais: Efeito da temperatura
}

(Mechanical model for the electro-conductivity in metals: temperature effects)

\author{
Vanderlei S. Bagnato ${ }^{1}$ e Vinícius Rodrigues ${ }^{2}$ \\ ${ }^{1}$ Instituto de Física de São Carlos, Universidade de São Paulo, São Carlos, SP, Brasil \\ ${ }^{2}$ Colégio Diocesano La Salle, São Carlos, SP, Brasil \\ Recebido em 28/10/2005; Aceito em 6/1/2006
}

\begin{abstract}
Condutividade elétrica é um dos conceitos mais importantes dentro dos aspectos da física moderna com grande extensão à ciência dos materiais. Ela é responsável por grande parte das aplicações de materiais metálicos e semicondutores. O entendimento de modelos microscópicos que reproduzem certas características, é um passo importante para o entendimento dos materiais. Neste trabalho damos continuidade ao uso de um sistema que se constitui em um análogo mecânico para entendermos como ocorrem as limitações de condutividade elétrica dos metais. Utilizando este modelo investigamos o efeito da temperatura na condutividade. O modelo aqui apresentado é bastante instrutivo e presta-se muito bem para demonstrações em sala de aula ou mesmo para realização de práticas de laboratórios em cursos de licenciatura ou práticas de ensino de Física no Ensino Médio. Palavras-chave: condutividade, modelo mecânico, modelo de Drude mecânico.
\end{abstract}

Using a mechanical analog of a solid we have demonstrated the Ohm's law showing the proportionality between the flux of current and the applied field. Introducing a vibrating motion into the device, the temperature effect was simulated showing the variation of the analog of conductivity with temperature for a normal conductor. The model is convenient for classroom demonstration of the Ohm's law as well as the variation with temperature.

Keywords: conductivity, mechanical model, Drude model.

\section{Introdução}

As propriedades elétricas dos materiais constituem importantes características que determinam suas aplicações. Dentro de um mundo movido basicamente pela eletricidade, o entendimento das características elétricas dos materiais, de forma clara é importante e serve para esclarecer estudantes e o público em geral de como ocorre a nível microscópico o fenômeno da resistência elétrica nos materiais condutores. Dentro da física, a conexão do mundo microscópico com o mundo macroscópico é um constante desafio. Normalmente procura-se realizar modelos que começam com a constituição básica da matéria nível atômico-molecular com o objetivo de reproduzir as propriedades macroscópicas do sistema. Dentro dos chamados efeitos elétricos, a condução de corrente por um determinado material condutor é um destes casos onde uma série de efeitos a nível atômico revelam-se através de uma grandeza macroscópica que é a resistência elétrica ou mais precisamente a condutividade dos materiais. Ao procurar-

\footnotetext{
${ }^{1}$ E-mail: vander@if.sc.usp.br.

Copyright by the Sociedade Brasileira de Física. Printed in Brazil.
}

mos explicar este fenômeno, normalmente temos que nos basear nas idéias básicas sobre a constituição da matéria e em especial o fato que para que elétrons caminhem pelo material, normalmente há vários obstáculos que funcionam como barreiras ao seu movimento. Esta é a essência microscópica para o surgimento da resistência elétrica.

O objetivo deste trabalho é mostrar os conceitos básicos da condutividade elétrica ( ou seu inverso a resistividade elétrica) dos metais através de um modelo mecânico que contém os principais efeitos presentes no sistema real. Através dele, pode-se ter um bom entendimento de vários fatores que influenciam a condutividade dos metais, como por exemplo, a temperatura. O modelo mecânico que apresentaremos aqui, já foi discutido anteriormente na Rev. Bras. Ens. Fis. [1] com relação aos seus aspectos básicos. Neste trabalho introduzimos um novo parâmetro, que consiste em mostrar que o modelo mecânico apresentado pode também simular a correta dependência da condutividade elétrica com a temperatura do sistema. 
Inicialmente apresentamos conceitos básicos sobre o tema, seguido de alguns detalhes da parte experimental e finalmente apresentamos os resultados obtidos.

\section{Lei de Ohm para a condução nos metais}

Uma das características importantes da condução nos metais é a chamada Lei de Ohm. Por meio desta lei, uma relação linear é estabelecida entre a voltagem aplicada no material e a corrente elétrica que nele surge. Uma outra forma de expressar esta importante lei é por meio do campo elétrico (e não a voltagem) e a densidade de corrente (corrente por unidade de área) fluindo no sistema. Vamos imaginar uma barra metálica, na qual é aplicada uma voltagem que resulta num campo elétrico $E$, constante ao longo da barra. O resultado da ação deste campo elétrico sobre as cargas móveis do metal é o aparecimento de uma densidade de corrente $J$, de tal maneira que o relacionamento entre estas grandezas resulta na chamada lei de Ohm [2]:

$$
J=\sigma E
$$

Nesta expressão, a constante de proporcionalidade $(\sigma)$ entre as grandezas envolvidas é chamada condutividade elétrica. Quanto maior é $\sigma$, maior é a densidade de corrente resultante da aplicação de um determinado campo elétrico. A Eq. (1) constitui a chamada lei de Ohm. Os materiais, para os quais essa relação é válida são chamados condutores ôhmicos. Há, no entanto muitos materiais que não obedecem esta relação e são denominados de não Ôhmicos.

A condutividade elétrica é uma característica bastante própria para materiais como os metais. Quanto mais facilmente os elétrons fluem quando submetidos a um campo elétrico, maior é a condutividade $\sigma$ do material.

Existem muitos efeitos a nível microscópico que contribuem para impedir o movimento dos elétrons num metal e, portanto limitam os valores possíveis para a condutividade. Dentre estes efeitos, está o fato de que, quando os elétrons são forçados a movimentaremse pela ação do campo elétrico externo, eles colidem com os íons (átomos) que constituem o material, transferindo a esses toda a energia cinética adquirida pela ação do campo elétrico. O resultado destas colisões é uma transferência de energia dos elétrons para o material como um todo, criando movimento dos átomos, e isto corresponde ao aquecimento do material (Efeito Joule).

Existe um modelo matemático simples que permite calcular $\sigma$ supondo uma rede de pontos com os quais os elétrons acelerados sofrerão colisões. Este é chamado de modelo de Drude [2]. Neste modelo, um gás de elétrons livres, sofre ação de um campo na presença de um arranjo de obstáculos, que correspondem aos átomos que constituem o metal. As colisões ocorrem em média a cada decorrência de um certo intervalo de tempo e em cada encontro toda a energia adquirida é transferida aos obstáculos. O modelo de Drude é essencialmente mecânico, e lida com trajetos de elétrons de tamanhos compatíveis com as dimensões atômicas.

\section{Um análogo mecânico macroscópico para a condutividade - Procedimento experimental}

É possível elaborar um análogo mecânico macroscópico deste modelo. Para isto, substituímos os átomos do metal por uma série de obstáculos mecânicos como pregos em uma tábua, e o campo elétrico é substituído pelo campo gravitacional. Este modelo já foi demonstrado anteriormente [1,3], e funciona muito bem revelando com fidelidade a dependência entre densidade de corrente e campo. Na Fig. 1 mostramos os aspectos básicos deste arranjo experimental.

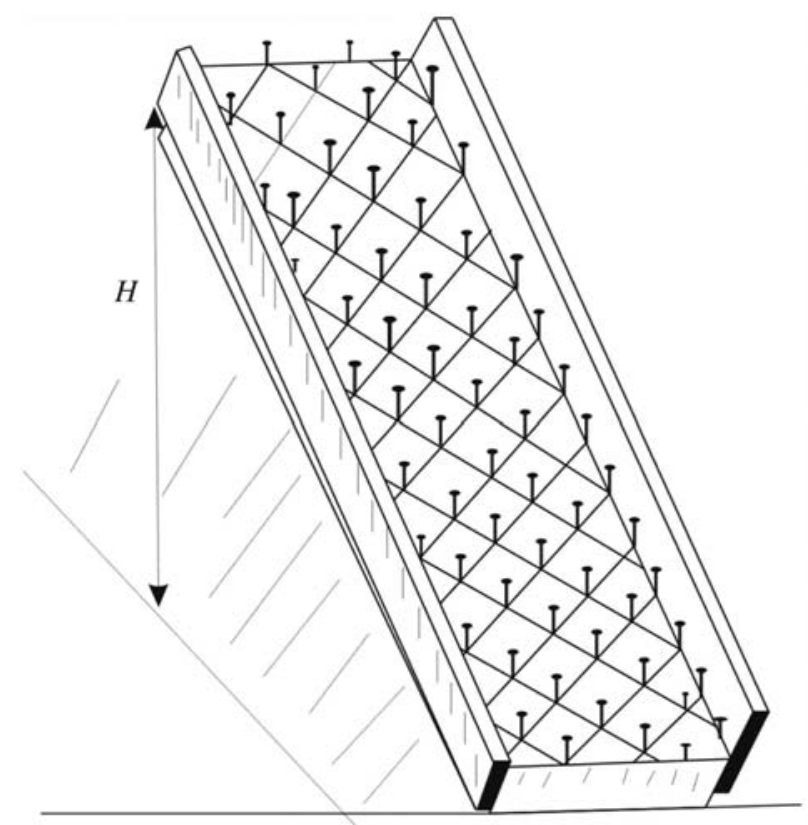

Figura 1 - Esquema mostrando o sistema experimental que permite fazer um análogo mecânico para a condutividade dos metais.

Imagine uma tábua na qual se colocam pregos, aleatoriamente distribuídos ou segundo uma rede préestabelecida (como é o caso apresentado na Fig. 1). Se esta tábua é inclinada criando uma diferença de potencial gravitacional entre seus extremos, uma bolinha de massa $m$ deixada no seu extremo superior, rola plano abaixo, sofrendo no seu caminho colisões com os pregos e sendo acelerada pelo campo gravitacional entre as colisões consecutivas. A situação é, portanto bem parecida com o modelo de Drude para a condução elétrica descrito em muitos textos básicos neste tema. Assim podemos dizer que é um análogo 
mecânico macroscópico para a condução elétrica. O equivalente do campo elétrico é agora a altura $H$, já que a componente paralela do campo gravitacional ao longo do plano da tábua será proporcional a esta altura $H$, que vai desde o extremo superior até o nível do extremo inferior. Também podemos pensar em termos da diferença de pontecial gravitacional, que nesta caso seria $m g H$. O fluxo de carga, que no caso elétrico, é a densidade de corrente, agora dever ser representado pelo fluxo de partículas que é dado por $1 / t_{m}$ onde $t_{m}$ é o tempo decorrido para a bolinha viajar entre os extremos da tábua. Assim, podemos definir o equivalente Mecânico da lei de Ohm, pela expressão:

$$
\frac{1}{t_{m}}=\sigma_{m} H
$$

Um gráfico $1 / t_{m}$ vs. $H$ fornece, portanto, uma reta cuja inclinação é a condutividade mecânica do sistema $\left(\sigma_{m}\right)$. Esta condutividade é equivalente à condutividade elétrica, e desta forma esta sujeita às mesmas variações desta. A Fig. 2, mostra uma imagem fotográfica do sistema durante a realização de uma medida onde pode-se observar a esfera de vidro descendo e colidindo com os pregos. O sistema é sustentado por quatro hastes que permitem a tábua vibrar paralela ao seu plano. Um motor colocado na parte inferior, possui uma placa fora de centro que ao girar força oscilações de todo sistema. A freqüência de oscilação é determinada pela rotação do motor (Fig. 3).

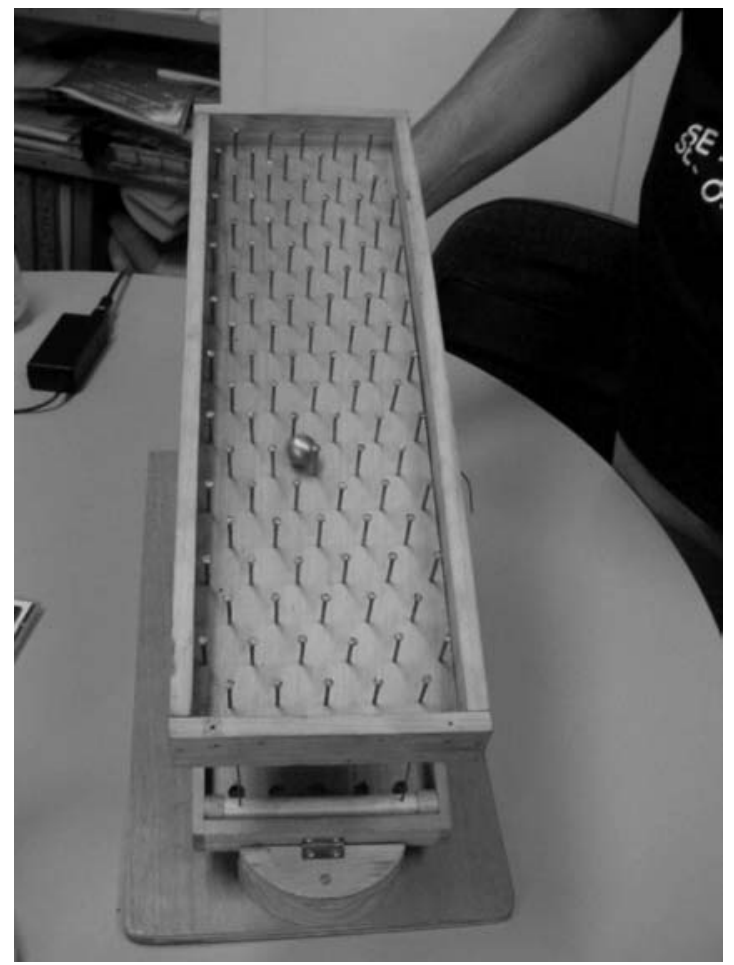

Figura 2 - Fotografia mostrando o sistema experimental durante a realização de uma medida.

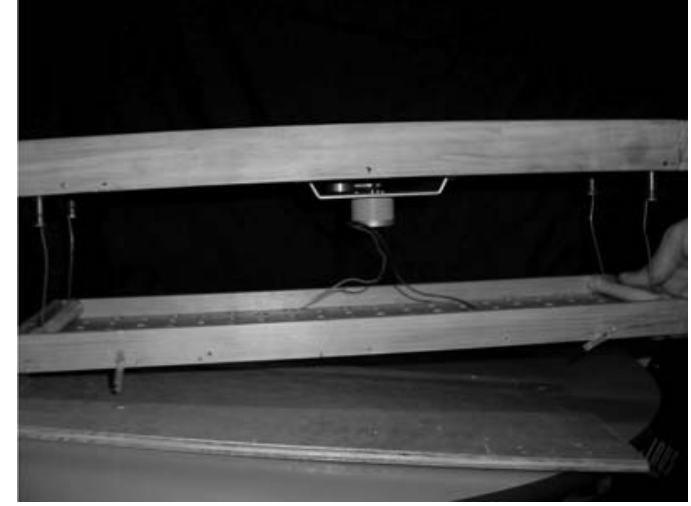

Figura 3 - Fotografia mostrando que o sistema montado é sustentado por quatro hastes que permite vibrações do plano. A própria elasticidade das hastes de aço fornecem o elemento elástico para as oscilações. Um motor fora de centro colocado na parte inferior, possui uma placa fora de centro que provoca oscilações. A freqüência de oscilação é determinada pela rotação do motor.

Neste sistema mecânico, a presença de oscilações simula o efeito da temperatura nos sólidos reais. Como sabemos a temperatura de um corpo é a medida da energia cinética dos seus constituintes atômicos. Aqui, como a amplitude de vibração é mantida praticamente constante, a freqüência representa a temperatura. Maior freqüência significa maior temperatura. Quando a freqüência é nula, significa a menor temperatura possível imposta ao sistema.

\section{Resultados observados}

O resultado de um dos experimentos realizados está mostrado na Fig. 4. A linearidade entre as grandezas consideradas permite obter, segundo a Eq. (2), um valor para a condutividade. Para este caso, os dados apresentados permitem obter $\sigma_{m} \approx(7,2 \pm 0,6) 10^{-4}$ $(\mathrm{s} \mathrm{mm})^{-1}$. A linearidade da curva da Fig. 4 mostra a validade do modelo e sua conexão com a lei de Ohm. É possível variar a densidade de pregos, criar distribuições aleatórias, criar diferentes redes, simular defeitos introduzindo pregos mais espessos e verificar as variações que tais mudanças causam na condutividade.

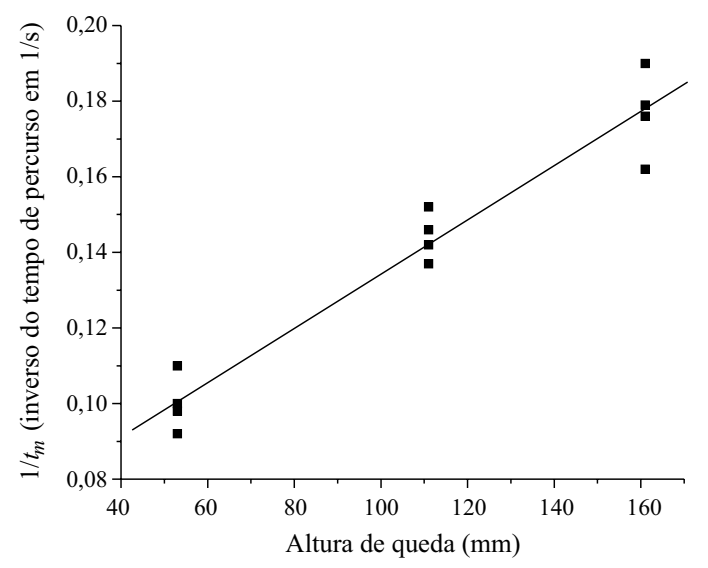

Figura 4 - Gráfico mostrando $1 / t_{m}$ vs. $H$. Para este caso cada ponto é o resultado da média de seis medidas. A condutividade mecânica é obtida pela inclinação da reta. 
É importante salientar que para cada altura $H$, deve-se repetir várias vezes o experimento, obtendo-se a média e o desvio padrão. O sistema é quantitativo e um bom uso da imaginação permite demonstrar para você mesmo ou para uma classe importantes conceitos relacionados com o tema. Um fato importante de ser salientado nos dados apresentados na Fig. 2 é que ao extrapolarmos $H \rightarrow 0$, não obtemos $1 / t_{m}=0$, como seria esperado pela Eq. (2). Os resultados experimentais são consistente com uma equação do tipo:

$$
\frac{1}{t_{m}}=\frac{1}{t_{0}}+\sigma_{m} H
$$

A existência do termo constante $1 / t_{0}$, já foi discutido anteriormente por Couto Tavares e colaboradores [3]. Este termo advém do fato que as colisões que ocorrem no modelo mecânico (bolinha-pregos) não são totalmente inelástica como assumidas no modelo de Drude. Ou seja, após cada colisão, ainda resta energia cinética na bolinha. Este fato, como mostra simulações numéricas realizadas na Ref. [3], leva à existência de um termo extra, tornando a Eq. (3) a forma mais correta de descrever o modelo.

\section{O efeito da temperatura na conduti- vidade mecânica}

É bastante conhecido o fato que a condutividade dos metais varia com a temperatura. A temperatura é uma grandeza macroscópica que representa a vibração dos átomos do metal e quanto maior é a temperatura, mais rápido eles vibram. Microscopicamente, a visão que temos é como que se fazendo os obstáculos vibrarem, torna-se mais difícil para os elétrons passarem, diminuindo a condutividade.

Para demonstrar o efeito da temperatura em nosso modelo mecânico, foi introduzido um sistema de suspensão como descrito que permitindo fazer a tábua com pregos vibrar em diferentes freqüências, preservando a amplitude de vibração. Considerando neste caso o equivalente mecânico da temperatura como sendo a freqüência com que toda a rede de pregos é agitada, pode-se investigar a variação da condutividade mecânica como função desta temperatura. Para cada freqüência de vibração $0 \mathrm{~Hz}, 1 \mathrm{~Hz}, 2 \mathrm{~Hz}, 3 \mathrm{~Hz}$, a condutividade mecânica foi medida.

A ausência de vibração é associada com a menor temperatura possível ao sistema, e associamos um aumento de temperatura mecânica igual à freqüência em Hz. Assim, obtivemos $\sigma_{m e c}$ para $\Delta T=0,1,2$ e 3 unidades arbitrarias de temperatura mecânica.

$\mathrm{O}$ resultado da variação de $\sigma_{m}$ com a temperatura está mostrado na Fig. 5.

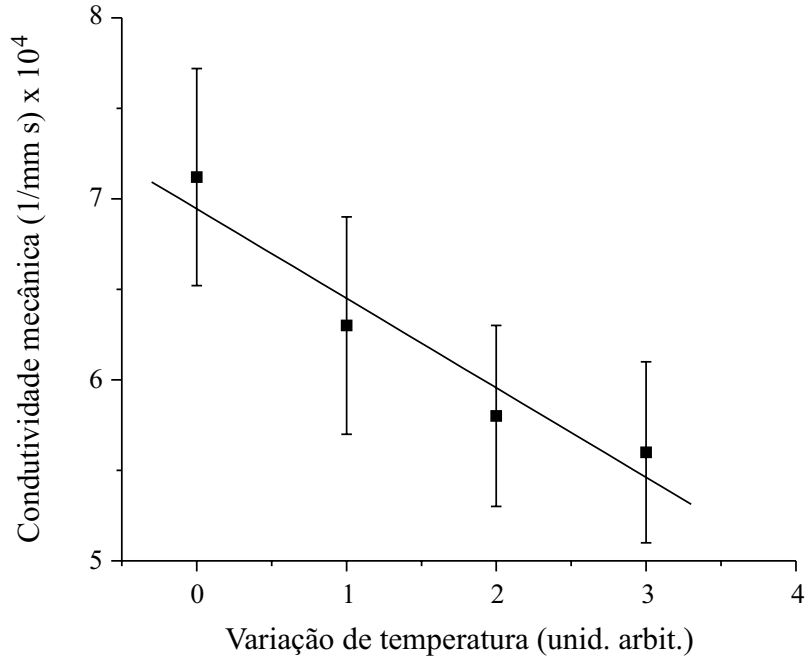

Figura 5 - Variação da condutividade mecânica como função do incremento da temperatura, associada ao aumento da freqüência de vibração do sistema mecânico.

Observa-se claramente a diminuição da condutividade mecânica com a temperatura. Nos vários livros textos [4] a dependência da condutividade com a temperatura é aproximadamente dada por:

$$
\sigma \sim \sigma_{0}\left[\frac{1}{1+A \Delta T}\right]
$$

onde $\sigma_{0}$ é uma constante que corresponde à condutividade a uma determinada temperatura referência, $A$ um coeficiente característico do material e $\Delta T$ é a variação de temperatura com relação a temperatura de referência. Como A é normalmente um coeficiente pequeno, podemos escrever a Eq. (4), aproximadamente como:

$$
\sigma_{m} \sim \sigma_{0}[1-A \Delta T] .
$$

Realizando um "fitting" dos dados experimentais com uma curva do tipo da Eq. (5), obtemos a linha colocada juntamente com os dados experimentais no gráfico da Fig. 5. Os valores característicos são $\sigma_{0}=6,9 \pm 0,5\left(\mathrm{~mm} \mathrm{~s}^{-1}\right.$ e $A=0,07 \pm 0,03(\mathrm{temp})^{-1}$. Estes valores numéricos são obviamente dependentes da estrutura específica de nosso modelo mecânico construído.

\section{Conclusões}

Com este trabalho demonstramos o equivalente mecânico da lei de Ohm, mostrando que a condutividade dos metais pode ser relativamente bem explicada por um modelo mecânico macroscópico. Além disto, o sistema montado é capaz de prever a influencia da temperatura na condutividade e serve como excelente sistema para despertar a intuição dos estudante com relação ao que ocorre a nível microscópico durante a produção de uma corrente elétrica. 


\section{Agradecimentos}

Agradecemos apoio do Grupo de Óptica e do Centro de Pesquisa em Óptica e Fotônica pelos investimentos feitos em difusão e ensino.

\section{Referências}

[1] V.S. Bagnato, Rev. Bras. Ens. Fís. 16, 129 (1994).
[2] O modelo de Drude pode ser encontrado nos livros de eletricidade de nível universitário como em D. Halliday e R. Resnick, Física (Livros Técnicos e Científicos, Rio de Janeiro, 1996), v. 3, $3^{\mathrm{a}}$ ed.

[3] M.C. Tavares, M. Fonte Boa and P.M. de Oliveira, Phys. Education 26, 195 (1991) e Phys. Education 27, 60 (1992).

[4] E.M. Purecell, Electricity and Magnetism (Berkeley Physics Course 2) (MacGraw-Hill, New York, 1965). 